Available online at GSC Online Press Directory

GSC Biological and Pharmaceutical Sciences

e-ISSN: 2581-3250, CODEN (USA): GBPSC2

Journal homepage: https://www.gsconlinepress.com/journals/gscbps

(SHORT COMMUNICATION)

\title{
Susceptibility of men to SARS-CoV-2 may be caused by sex differences in ACE2 expression
}

\author{
Hayashi Takuma ${ }^{1,}{ }^{*}$, Mandan Masaki ${ }^{2}$, Yaegashi Nobuo ${ }^{3}$ and Konishi Ikuo 1, 2, 4 \\ ${ }^{1}$ National Hospital Organization Kyoto Medical Center, Kyoto, Kyoto, Japan. \\ ${ }^{2}$ Department of Obstetrics and Gynecology, Kyoto University School of Medicine, Kyoto, Japan. \\ ${ }^{3}$ Department of Obstetrics and Gynecology, Tohoku University School of Medicine, Miyagi, Japan \\ ${ }^{4}$ Immediate Past President, Asian Society of Gynecologic Oncology, Tokyo, Japan.
}

Publication history: Received on 22 July 2020; revised on 02 August 2020; accepted on 03 August 2020

Article DOI: https://doi.org/10.30574/gscbps.2020.12.2.0235

\begin{abstract}
Experts have noted important aspects regarding the clinical symptoms of coronavirus disease 2019 (COVID-19), which is caused by severe acute respiratory syndrome coronavirus type 2 (SARS-CoV-2). For instance, the clinical symptoms are clearly different between the sexes. Men have higher SARS-CoV-2 infection rates than women. COVID-19 symptoms are also likely to be more severe in men than in women. Consequently, SARS-CoV-2-infected men experience fatal complications. The research results of medical experts highlight two possible reasons for the difference in COVID-19 clinical symptoms between the sexes: the risk derived from lifestyle patterns and the biological (immune response) risk. However, with regard to COVID-19, researchers are still unable to answer many questions regarding why the infection and fatality rates of men are higher than those of women. In this review, the authors report differences in blood levels of angiotensin-converting enzyme 2 as a reason for the differences between the sexes seen in COVID-19 symptoms including the most up-to-date information.
\end{abstract}

Keywords: ACE2; Binding region; COVID-19; Severe acute respiratory syndrome coronavirus 2

\section{Introduction}

At deCODE Genetics-Amgen in Iceland, Daniel F. Gudbjarsson et al. examined and reported the genomic features of severe acute respiratory syndrome coronavirus type 2 (SARS-CoV-2) and the epidemiological features of coronavirus disease 2019 (COVID-19). These findings were based on specific high-risk populations in Iceland and a resident-based survey conducted among citizens. At the end of February 2020, a patient in Iceland was first diagnosed with COVID-19. Unfortunately, the data were very limited to reveal how SARS-CoV-2, which is the causative agent of COVID-19, infects and spreads among Icelandic people. The researchers reported that SARS-CoV-2 haplotypes are diverse and change over time. Furthermore, the prevalence of SARS-CoV-2 infection in Iceland is lower in children aged under 10 years and women than in those aged over 10 years and men. Nevertheless, during the screening period, the prevalence of infection in the general population did not change substantially. Hence, the containment measures against COVID-19 in Iceland are seemingly effective.

The new infectious disease COVID-19 develops when an individual is infected with SARS-CoV-2, which is a newly discovered virus strain [1]. It remains unclear whether SARS-CoV-2 has occurred from any kind of infection circumstances or whether it came to infect humans. The onset of COVID-19 was first reported in people working in a seafood market in Wuhan, China. Subsequently, SARS-CoV-2 was also detected in the wildlife trading area in Wuhan [2].

\footnotetext{
* Corresponding author: Hayashi Takuma
} 
Therefore, the cause of COVID-19 is lurking within Wuhan [3].

SARS-CoV-2 can infect individuals of all ages and from different walks of life [4]. Therefore, we need to act while thinking that we might be infected with SARS-CoV-2. Clearly, COVID-19 will have a significant impact on people's lifestyles. Globally, SARS-CoV2 infection has led to a shortage in medical equipment and personnel; consequently, patients with severe symptoms may not receive adequate treatment. Social lockdown has been implemented in various affected regions to contain the spread of infection (e.g., closing of nonessential business establishments); as a result, their economy has plummeted, causing an upsurge in unemployment rates, and eventually, the inability of employees to meet basic needs, such as food. Under these circumstances, disparities among people and distortions in social structure occur. As research on COVID-19 progresses, "sex" and "age" have been highlighted as the causes of "differences" in health risk among infected individuals [5].

In China and Italy, studies have compared the mortality rate of COVID-19 between men and women. Results showed that men have a higher mortality rate than women [6]. Hence, the background of the difference in mortality between men and women was examined. Results showed that smoking status and female hormones seem to affect the mortality rate of COVID-19.

\section{Investigation of the actual status of COVID-19 by conducting polymerase chain reaction (PCR) tests on specific populations and those with a high infection risk}

Daniel F. Gudbjarsson et al. from deCODE Genetics-Amgen in Iceland conducted a detailed analysis of two SARS-CoV-2 tests (examination of a specific group of individuals who are at a high risk of infection and examination of publicly recruited groups for group screening) [7].

Since January 31, 2020, residents of Iceland who have a high risk of infection to SARS-CoV-2 (individuals with symptoms such as cough, fever, arthralgia, and shortness of breath; individuals who had recently returned from high-risk countries/regions; or individuals who had contact with infected individuals) (specific population groups) have been examined [7]. In addition, since March 13, 2020, residents with asymptomatic or mild cold symptoms (open recruitment group) have been examined. Specifically, the online registration and examination of the target population have been started [7].

In April 4, 2020, Iceland's infection rate was 0.6\%, and the positive rate for children aged under 10 years and women was low [7]. From January 31, 2020, to March 31, 2020, 1,221 of 9,199 (13.3\%) individuals in the specific population group tested positive for SARS-CoV-2 [7]. Of 10,797 (0.8\%) individuals from the mass screening group who were tested from March 13 to April 1, 87 tested positive for SARS-CoV-2 [7]. In the randomized group tested between April 1 and 4 , 13 of 2,283 individuals (0.6\%) tested positive for the virus [7]. Overall, $6 \%$ of Iceland's population was examined.

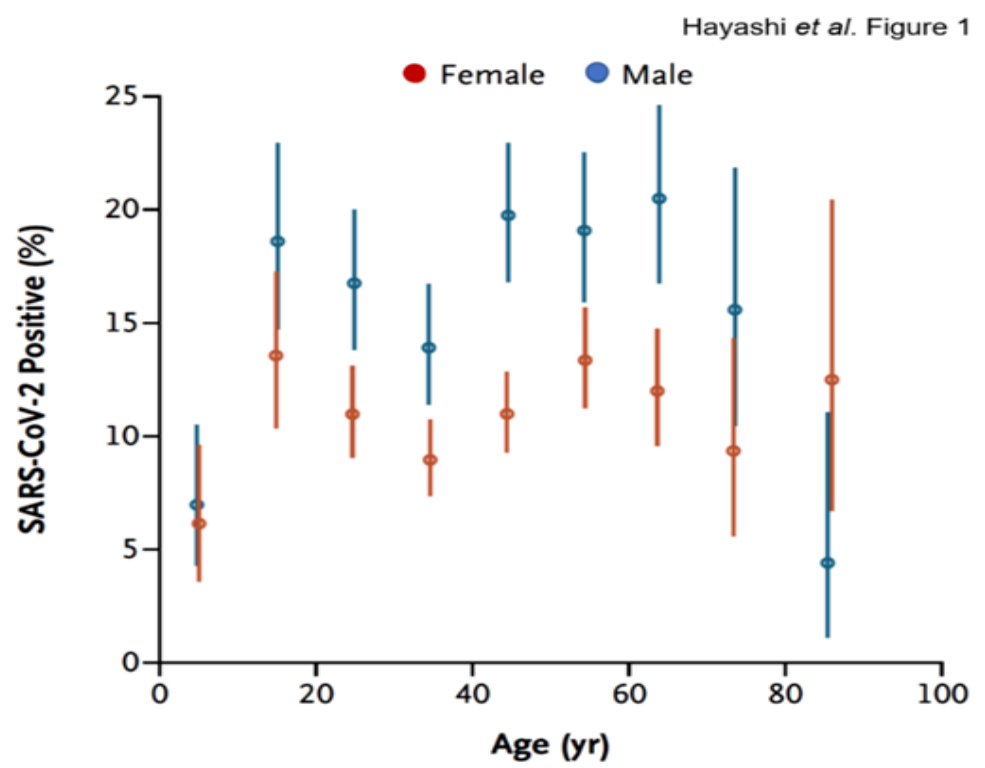


Figure 1 Distribution of targeted testing and mass screening for SARS-CoV-2 and percentages of positive results according to age and sex

Research findings shown are the percentages of participants who tested positive stratified according to sex in the targeted testing group. The figure is adapted from Reference 7.

The mean age of individuals was 40.0 years in the early group of the specified population group, 40.4 years in the late group of the specified group, 38.6 years in the open recruitment group, and 45.4 years in the randomized group [7]; for the SARS-CoV-2-positive patients, the mean age was $44.4,41.3,40.8$, and 50.5 years in these groups, respectively [7]. In the specific population group, the number of individuals with SARS-CoV-2 infection aged 10 years or older $(1,183 / 8,635$ cases, $13.7 \%$ ) was higher than that of infected individuals aged under 10 years (38/564 cases, 6.7\%) [7]. This tendency was similar in the mass screening group (0\%, aged less than 10 years; $0.8 \%$, aged over 10 years). In terms of sex, men had a higher rate of SARS-CoV-2 infection than women (Figure 1).

In the specific population group, the positive rate in women was $11.0 \%$, whereas that in men was $16.7 \%$ (odds ratio [OR]: 1.66, 95\% confidence interval [CI]: 1.47-1.87), and in the mass screening group, the positive rate in women was $0.6 \%$, whereas that in men was $0.9 \%$ (OR: $1.55,95 \%$ CI: $1.04-2.30$ ) [7].

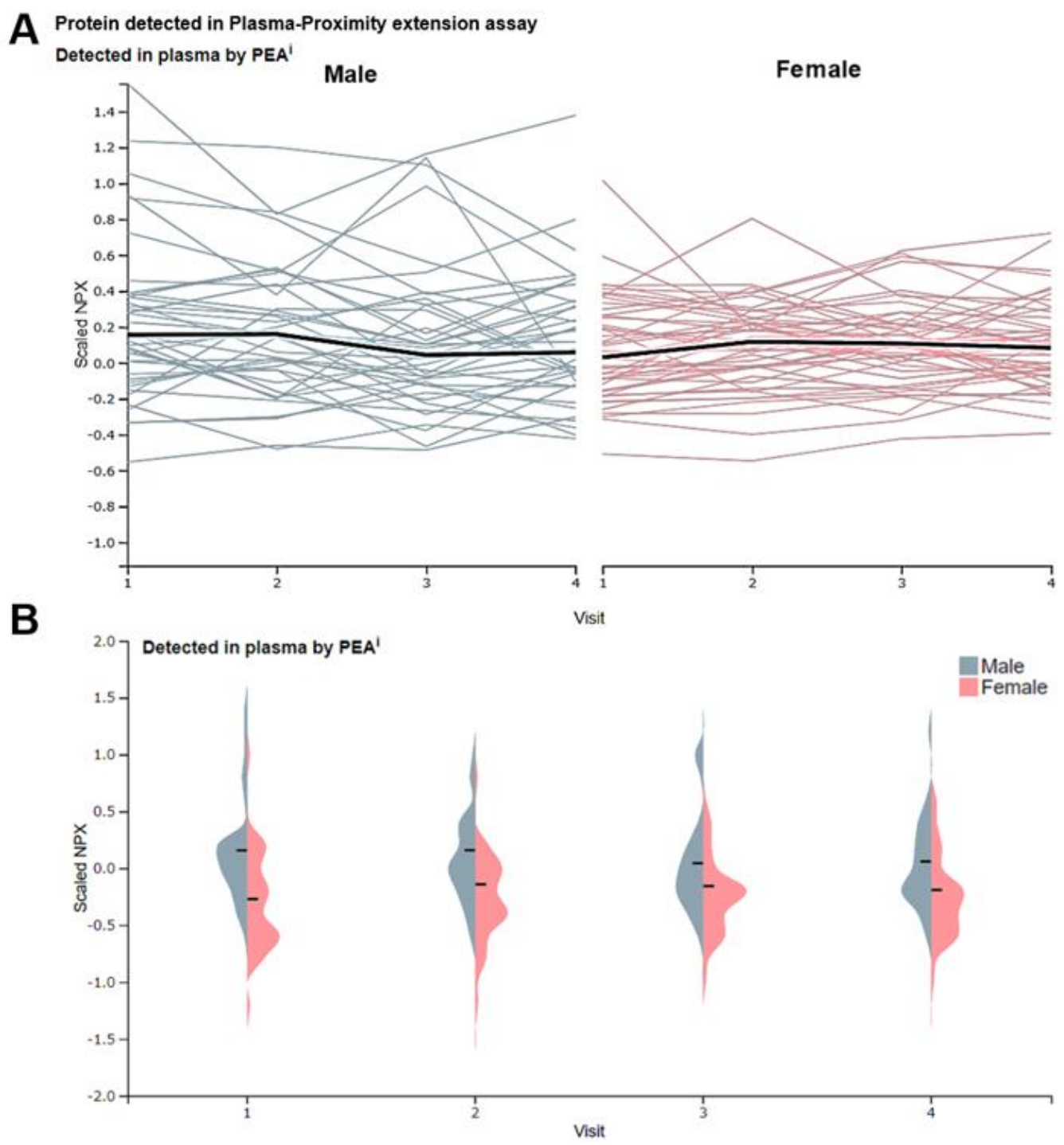

Figure 2 ACE2 protein expression detected using the plasma-proximity extension assay

The ACE2 concentration in plasma derived from healthy humans was measured by the plasma-proximity extension assay note1. The plasma ACE2 concentration of each subject was converted into a relative value. A The relative values 
of ACE2 concentration of each subject were plotted according to sex. B In addition, the relative ACE2 concentration was plotted according to sex, and the average is shown. These results showed that the plasma ACE2 concentration (relative value) was clearly higher in men than in women.

In two cohort populations, the positive rate of SARS-CoV-2 infection in men was higher than that in women. SARS-CoV2 infects host cells, with angiotensin-converting enzyme 2 (ACE2) as the receptor. Specifically, the first step in the process of SARS-CoV-2 infection is the recognition and binding of ACE2 by the spike glycoprotein of SARS-CoV-2. Thus, our research group examined the difference in ACE2 expression levels in vivo between men and women. Our research group measured the plasma ACE2 protein levels of men and women using the plasma-proximity extension assay note1. Furthermore, the plasma ACE2 concentration of each subject was converted into a relative value. The relative values of ACE2 concentration of each subject were plotted according to sex (Figure 2A). The relative ACE2 concentration was also plotted by sex, and the average was calculated (Figure 2B). Research results showed that the plasma ACE2 concentration (relative value) was clearly higher in men than in women (Figure 2A, B). It is probable that ACE2 is more highly expressed in the male body than in the female body. Therefore, our findings suggest that men are more susceptible to COVID-19 than women.

\section{Widespread variety of SARS-CoV-2 haplotypes}

SARS-CoV-2 genomic RNA extracted from 643 samples was sequenced; the results revealed that SARS-CoV-2 haplotypes were diverse and varied over time. In other words, the SARS-CoV-2 haplotype composition in the initial stage of the specific population group is different from that of the mass screening group [7]. These findings suggest that in the early stage, most of the SARS-CoV-2 haplotypes observed in Iceland originated in Italy and Austria [7]. However, in the mass screening group after mid-March, the type of SARS-CoV-2 found in Iceland was the haplotype of SARS-CoV-2 found in the UK [7].

On mass screening, the positive rate of SARS-CoV-2 infection remained unchanged for 20 days [7]. The rates of SARSCoV-2 infection did not differ significantly between the open recruitment group and the randomized group. Therefore, the haplotype of the gene mutation of SARS-CoV-2 was unlikely to be responsible for the increased viral susceptibility in men. In countries with COVID-19 cases such as Italy and China, the number of deaths tends to be higher in men than in women. COVID-19 experts have emphasized that the reason for this trend is men's poor general health caused by smoking and drinking [8]. According to global statistics in 2015, the number of male smokers was approximately five times that of female smokers [9], consistent with alcohol consumption among men [10]. Many studies have confirmed the impact of severe COVID-19 toward unhealthy lifestyle and chronic disease.

The severe acute respiratory syndrome (SARS) and the Middle East respiratory syndrome, which have been previously prevalent, tended to have a higher mortality rate in men than in women $[11,12]$. Biologically, the immune response to viral infections was stronger in women bearing children than in men [12]. Sex-based immunity is associated with high survival rates. Furthermore, COVID-19 is more likely to cause a severe health status in patients with underlying conditions, such as hypertension, cardiovascular disease, and chronic lung disease [13]. The current survey in Iceland revealed that men with these conditions are clearly higher in number than women.

According to the Italian National Institutes of Health situation report, smokers who tested positive for SARS-CoV-2 infection had more than $30 \%$ more severe cases than nonsmokers $[14,15]$. The number of smokers in need of intensive care and ventilator is more than two-fold that of nonsmokers. In Italy, men have a higher rate of hypertension than women of the same age group. Moreover, Chinese men have more hypertension cases and type 2 diabetes cases than Chinese women. The clinical symptoms of COVID-19 are clearly different between the sexes. Men have a higher rate of SARS-CoV-2 infection than women. Currently, numerous research results regarding the sex difference of SARS-CoV-2 infection rate have been reported. The cause of the sex differences observed in COVID-19 may not be the manifestation of single factors, such as sex hormones, but polygenic factors in which multiple factors cross intricately. To gain further clarity on issues regarding COVID-19, we need to perform further clinical studies.

\section{Conclusion}

There was an obvious difference in clinical symptoms between the sexes in COVID-19. Men have higher rates of SARS$\mathrm{CoV}-2$ infection than women. Based on current research results, various evidences have been reported regarding the sex difference in the infection rate of SARS-CoV-2. In this review, differences in blood ACE2 levels between males and females were reported as one of the causes of various differences between the sexes in COVID-19. Further clinical studies should be conducted to determine the sex differences in COVID-19. 


\section{Footnote}

Plasma-proximity extension assay note1: Olink-based proximity extension assays were performed for 748 proteins based on a longitudinal wellness study including 86 individuals with four visits in 1 year at 3-month intervals. The results are presented as normalized protein expression for the individual genes corresponding to these proteins for all men and women separately.

Below is a summary of the variation among individuals and within each individual of these 748 proteins calculated as the mean standard variation (SD) for each protein within each visit and across all analyzed individuals $(\mathrm{n}=86)$ and as the mean SD for each protein within each individual across all visits $(n=4)$.

\section{Data Sharing}

Data are available on various websites and have been made publicly available (more information can be found in the first paragraph of the Results section).

\section{Compliance with ethical standards}

\section{Acknowledgments}

We thank Professor Richard A. Young (Whitehead Institute for Biomedical Research, Massachusetts Institute of Technology, Cambridge, MA) for his research assistance. This study was supported in part by grants from the Japan Ministry of Education, Culture, Science, and Technology (No. 24592510, No. 15K1079, and No. 19K09840), the Foundation of Osaka Cancer Research, The Ichiro Kanehara Foundation for the Promotion of Medical Science and Medical Care, the Foundation for Promotion of Cancer Research, the Kanzawa Medical Research Foundation, The Shinshu Medical Foundation, and the Takeda Foundation for Medical Science.

\section{Disclosure of conflict of interest}

The authors declare no potential conflicts of interest. The funders had no role in study design, data collection and analysis, decision to publish, or preparation of the manuscript.

\section{Statement of informed consent}

Informed consent was obtained from all individual participants included in the study.

\section{References}

[1] World Health Organization. (WHO) Novel Coronavirus (2019-nCoV) Situation Report-21.

[2] Wang FS and Zhang C. (2020). What to do next to control the 2019-nCoV epidemic? Lancet, 395(10222), 391393.

[3] Zhu N, Zhang D, Wang W, Li X, Yang B, Song J, Zhao X, Huang B, Shi W, Lu R, Niu P, Zhan F, Ma X, Wang D, Xu W, Wu G, Gao GF and Tan W. (2020). China Novel Coronavirus Investigating and Research Team. A Novel Coronavirus from Patients with Pneumonia in China, 2019. New England Journal of Medicine, 382(8), $727-733$.

[4] Liu W, Zhang Q, Chen J, Xiang R, Song H, Shu S, Chen L, Liang L, Zhou J, You L, Wu P, Zhang B, Lu Y, Xia L, Huang L, Yang Y, Liu F, Semple MG, Cowling BJ, Lan K, Sun Z, Yu H and Liu Y. (2020). Detection of Covid-19 in Children in Early January 2020 in Wuhan, China. New England Journal of Medicine, 382(14), 1370-1371.

[5] Sokolowska M, Lukasik Z, Agache I, Akdis CA, Akdis D, Akdis M, Barcik W, Brough H, Eiwegger T, Eliaszewicz A, Eyerich S, Feleszko W, Gomez Casado C, Hoffmann-Sommergruber K, Janda J, Jiménez-Saiz R, Jutel M, Knol E, Kortekaas Krohn I, Kothari A, Makowska J, Moniuszko M, Morita H, O'Mahony L, Nadeau K, Ozdemir C, Pali-Schöll I, Palomares O, Papaleo F, Prunicki M, Schmidt-Weber CB, Sediva A, Schwarze J, Shamji MH, Tramper-Stranders G, van W, de Veen and Untersmayr E. (2020). Immunology of COVID-19: mechanisms, clinical outcome, diagnostics and perspectives - a report of the European Academy of Allergy and Clinical Immunology (EAACI). Allergy.

[6] World Health Organization. ((2020 .Gender and COVID-19: advocacy brief, 14 May 2020. World Health Organization. 
[7] Gudbjartsson DF, Helgason A, Jonsson H, Magnusson OT, Melsted P, Norddahl GL, Saemundsdottir J, Sigurdsson A, Sulem P, Agustsdottir AB, Eiriksdottir B, Fridriksdottir R, Gardarsdottir EE, Georgsson G, Gretarsdottir OS, Gudmundsson KR, Gunnarsdottir TR, Gylfason A, Holm H, Jensson BO, Jonasdottir A, Jonsson F, Josefsdottir KS, Kristjansson T, Magnusdottir DN, le Roux L, Sigmundsdottir G, Sveinbjornsson G, Sveinsdottir KE, Sveinsdottir M, Thorarensen EA, Thorbjornsson B, Löve A, Masson G, Jonsdottir I, Möller AD, Gudnason T, Kristinsson KG, Thorsteinsdottir U and Stefansson K. (2020). Spread of SARS-CoV-2 in the Icelandic Population. New England Journal of Medicine, 382(24), 2302-2315.

[8] Nguyen HT, Do BN, Pham KM, Kim GB, Dam HTB, Nguyen TT, Nguyen TTP, Nguyen YH, Sørensen K, Pleasant A and Duong TV. (2020). Fear of COVID-19 Scale-Associations of Its Scores with Health Literacy and Health-Related Behaviors among Medical Students. Int J Environ Res Public Health, 17(11), 4164.

[9] Gender equality is good for health. Facts on gender and tobacco- World Health Organization.

[10] Hung CC, Chang HY, Luh DL, Wu CC and Yen LL. (2015). Do parents play different roles in drinking behaviours of male and female adolescents? A longitudinal follow-up study. BMJ Open, 5(4), e007179.

[11] Möhn N, Pul R, Kleinschnitz C, Prüss H, Witte T, Stangel M and Skripuletz T. (2020). Implications of CoVID-19 Outbreak on Immune Therapies in Multiple Sclerosis Patients-Lessons Learned From SARS and MERS. Frontiers in immunology, 11, 1059.

[12] Kloc M, Ghobrial RM and Kubiak JZ. (2020). The Role of Genetic Sex and Mitochondria in Response to COVID-19 Infection. International archives of allergy and immunology, 181, 629-634.

[13] Pettit NN, MacKenzie EL, Ridgway J, Pursell K, Ash D, Patel B and Pho MT. (2020). Obesity is Associated with Increased Risk for Mortality Among Hospitalized Patients with COVID-19. Obesity (Silver Spring).

[14] Wang Z, Zou Z, Yang Z, Dong Y and Ma J. (2017). Association between exposure to the Chinese famine during infancy and the risk of self-reported chronic lung diseases in adulthood: a cross-sectional study. BMJ Open, 7(5), e015476.

[15] Guo FR. (2020). A flaw on a meta-analysis of smoking and the severity of COVID-19: the association should have been endorsed. Journal of public health (Oxford, England), fdaa083.

\section{How to cite this article}

Hayashi T, Mandan M, Yaegashi N and Konishi I. (2020). Susceptibility of men to SARS-CoV-2 may be caused by sex differences in ACE2 expression. GSC Biological and Pharmaceutical Sciences, 12(2), 01-06. 